\title{
PROJECTIVE STRUCTURES ON AN ANNULUS AND HANKEL FORMS
}

\author{
by JAAK PEETRE and GENKAI ZHANG
}

(Received 14 February, 1990)

0. Introduction. A general theory of Hankel forms over domains in one or several variables has been set forth in [6]. In [7] the study of Hankel forms over an annulus in the complex plane $\mathbb{C}$ was begun. (An extension of the results of [7] to multiply connected domains was given in [4].) The present paper amplifies the results of [7] in various respects. First of all we define and study more general Hankel forms associated with a one parameter family of projective structures on the annulus. This displays several new features. For instance, we are now dealing with quadratic integral metrics which do not correspond to integration of the square of the function with respect to a weight. Furthermore, whereas in [7] essentially only the issue of the boundedness of Hankel forms was studied, we obtain here rather satisfactory $S_{p}$-results, even for $0<p<1$. The question which remains is, of course, to which extent all this extends to multiply connected domains (or more general (open) Riemann surfaces).

The plan of the paper is roughly the following.

Section 1 gives preliminaries on projective structures in general.

In Section 2 we then specialize to the case of an annulus $\Omega=\Omega_{R}=\{z: 1<|z|<R\}$. In particular, we single out a one parameter family of projective structures on $\Omega$. To any of these projective structures and an integer "order" $\alpha \geq 0$ there corresponds an integral metric. Up to equivalence of norm these spaces for a given $\alpha$ do not depend on the projective structure and we get the usual Dzhrbashyan (or weighted Bergman) spaces $A^{\alpha, 2}=A^{\alpha, 2}(\Omega)$.

In Section 3 we study the boundedness of the corresponding Hankel forms.

Section 4 is devoted to the $S_{p}$-theory, $1 \leq p<\infty$.

In Section 5 we prove a decomposition theorem for the spaces $A^{\alpha, 2}(\Omega)$. In this as well as in the previous Section we largely follow the general scheme layed down in the work of Rochberg (cf. [11], [12]).

With the aid of the latter we can then in Section 6 extend the results of Section 4 to the case $0<p<1$. However, for technical reasons we require here the restriction $\alpha+2>1 / p$.

Finally, in the last Section we study the case when the order $\alpha$ is non-integer $(>-1)$. Here our result are somewhat less complete.

Note that in order to keep the paper within reasonable size, we have been forced to omit many technical details. Thus, some of our proofs are mere sketches.

1. Preliminaries on projective structures. Let us say a few words about projective structures in general. Let $\Omega$ be any Riemann surface. A projective structure on $\Omega$ is given by a covering of $\Omega$ by open subsets denoted $U, U^{\prime}, \ldots$ together with corresponding coordinate functions denoted $\zeta, \zeta^{\prime}, \ldots$ in the overlap $U \cap U^{\prime}$ of any two of these open subsets it is assumed that the corresponding coordinate functions $\zeta, \zeta^{\prime}$ are related by a fractional linear transformation, i.e. $\zeta^{\prime}=\frac{a \zeta+b}{c \zeta+d}$ with $a d-b c=1$.

Glasgow Math. J. 33 (1991) 247-266. 
REMARK. For the reader's convenience we add here also a few informal words concerning the language of holomorphic line bundles. We say that we have a holomorphic line bundle $\xi$ over our Riemann surface $\Omega$ if there is given a covering of $\Omega$ by open subsets denoted $V_{1}, V_{2}, \ldots$ and non-vanishing holomorphic functions $g_{12}, \ldots$ ("transition functions") defined in the overlaps $V_{1} \cap V_{2}, \ldots$; it is required that $g_{12} g_{23}=g_{13}, \ldots$ In other words, these data determine an element of the cohomology group $H^{1}\left(\Omega, O^{\times}\right)$, where $\mathcal{O}^{\times}$is the sheaf of germs of non-vanishing holomorphic functions on $\Omega$. In particular, it follows that any two holomorphic line bundles can be multiplied with each other, which again amounts to multiplication of the respective transfer functions. The fiber $\xi_{z}$ of $\xi$ at a point $z \in \Omega$ consists of all collections of complex numbers $x_{1}, x_{2}, \ldots$, which are related by relations $x_{1}=g_{12}(z) x_{2}, \ldots$ Thus a (holomorphic) section $s$ of $\xi$ is determined by a family of holomorphic functions $s_{1}, s_{2}, \ldots$, defined in $V_{1}, V_{2}, \ldots$ respectively, such that $s_{1}=g_{12} s_{2}, \ldots$ in the overlaps $V_{1} \cap V_{2}, \ldots$ As an example of a holomorphic line bundle, we mention the canonical bundle $\kappa$ of $\Omega$. In this case, we can take the covering $V_{1}, V_{2}, \ldots$ to be coordinate neighborhoods $z_{1}, z_{2}, \ldots$; if any two coordinates $z_{1}$ and $z_{2}$ are related by an equation $z_{1}=\varphi\left(z_{2}\right)$ we take the corresponding transfer function to be $g_{12}=\varphi^{\prime}$. If we consider $\kappa^{2}$ then we have instead to take $g_{12}=\left(\varphi^{\prime}\right)^{2}$, and similarly for higher powers $\kappa^{m}$. Sections of $\boldsymbol{K}^{m}$ are known as (differential) forms of degree $m$. For more details concerning these matters see e.g. [2].

Continuing the main discussion, a general method of generating projective structures is to give a second order differential operator on $\Omega$. Let it be given, in terms of a general local coordinate $z$, by $L=\frac{d^{2}}{d z^{2}}+q(z)$. If $\eta_{1}$ and $\eta_{2}$ are any two independent null solutions, then a projective coordinate is obtained by setting $\zeta=\frac{\eta_{1}}{\eta_{2}}$. We agree that solutions of $L \eta=0$ transform like sections of the inverse of a spin bundle $\lambda$ over $\Omega$ (a square root of $\kappa$, so that $\kappa^{2}=\lambda$ ). If $w$ is another local coordinate related to $z$ by the equation $z=\varphi(w)$, then $\theta(w)=\eta(\varphi(w)) \varphi^{\prime}(w)^{-1 / 2}$ is in the kernel of the operator $L_{1}=$ $\frac{d^{2}}{d w^{2}}+q_{1}(w)$, where

and

$$
q_{1}(w)=q(w)\left(\varphi^{\prime}(w)\right)^{2}+\{\varphi, w\}
$$

$$
\{\varphi, w\} \stackrel{\text { def }}{=} \frac{1}{2} \varphi^{\prime}(w)^{1 / 2} \frac{d}{d w}\left[\varphi^{\prime}(w)^{-3 / 2} \varphi^{\prime \prime}(w)\right] \quad \text { (Schwarz derivative) }
$$

The operator $L$ may be viewed as the second Bol operator. One can also introduce Bol operators of higher order. The null solutions to the $\mu$ th order Bol operator $L_{\mu}$ are, in terms of the projective coordinate $\zeta$, precisely $1, \zeta, \zeta^{2}, \ldots, \zeta^{\mu-1}$, and, in terms of $z$, they are $\eta_{2}^{\mu-1}, \eta_{2}^{\mu-2} \eta_{1}, \ldots, \eta_{1}^{\mu-1}$. Now they may be viewed as sections of $\lambda^{1-\mu}$. Thus the operator reads

$$
\frac{d^{\mu}}{d \zeta^{\mu}} \text { respectively } \frac{d^{\mu}}{d z^{\mu}}+A_{2} \frac{d^{\mu-2}}{d z^{\mu-2}}+\ldots+A_{\mu}
$$

where $A_{2}, \ldots, A_{\mu}$ are certain "universal" polynomials in $q$ and its derivatives [3]. It is clear that $L_{1} \equiv d$ and $L_{2} \equiv L$. 
From the above it is clear that the projective structures on a given Riemann surface $\Omega$ form an affine space over the vector space of quadratic differentials (sections of $\boldsymbol{K}^{2}$ ). ${ }^{1}$ If we have two different second order Bol operators then their difference is a quadratic differential. We obtain a vector space by selecting as base point the projective structure which corresponds to the universal cover $\tilde{\Omega}$ of $\Omega$.

EXAmple 1. Assume that $\Omega$ is compact of genus $g$. Then this space is finite dimensional. Denoting its (finite) dimension by $d$ we have (in view of the Riemann-Roch theorem)

$$
d= \begin{cases}0 & \text { if } g=0 \\ 1 & \text { if } g=1 \\ 3 g-3 & \text { if } g>1\end{cases}
$$

2. Projective structures on an annulus. Integral metrics. Let now $\Omega$ be the annulus $\Omega=\{z: 1<|z|<R\}$ in the complex plane $\mathbb{C}$. It is natural to consider together with it the universal cover $\tilde{\Omega}$ of its Schottky double $\hat{\Omega}$. This amounts to introducing a "uniformizing" parameter $u$ given by $z=e^{-i u}$. We are interested in those projective structures on $\Omega$ which come from this universal cover. We know (Section 1) that a projective structure on any Riemann surface is determined by its second Bol operator $L_{2}$. In the case at hand we thus have $L_{2}=\frac{d^{2}}{d u^{2}}+q(q=$ constant). The corresponding null solutions are (unless $q=0$ ) $\eta_{1}=e^{\sqrt{-q u}}$ and $\eta_{2}=e^{-\sqrt{-q u}}$. It follows that the corresponding uniformizing parameter is

$$
\zeta=\frac{\eta_{1}}{\eta_{2}}=e^{2 \sqrt{-q u}}
$$

EXAMPLE 2. The universal cover $\tilde{\Omega}$ of $\Omega$ itself corresponds to

$$
\zeta=z^{i \pi / \log R}=e^{i \pi \log z / \log R}=e^{i \pi(-i u) / \log R}=e^{\pi u / \log R} .
$$

It follows that in this case

$$
2 \sqrt{-q}=\frac{\pi}{\log R} \quad \text { or } \quad q=-\frac{\pi^{2}}{4(\log R)^{2}}
$$

EXAMPLE 3. Consider the projective structure corresponding to the "circular" realization of $\Omega$ (=the given one). Then the Bol operator is, using $z$ as a parameter, $L_{2}=\frac{d^{2}}{d z^{2}}$ or, passing to $u, L_{2}=\frac{d^{2}}{d u^{2}}+\frac{1}{4}$, that is, $q=\frac{1}{4}$.

EXAmple 4. The case $q=0$ corresponds to the universal cover of the double.

The higher order Bol operators are, using the parameter $u$, given by

$$
L_{\mu}=\prod_{k=0}^{\mu-1}\left[\frac{d}{d u}-\sqrt{-q}(\mu-1-2 k)\right] .
$$

\footnotetext{
${ }^{1}$ Strictly speaking, this formulation is not entirely correct. To render this statement correct we must interpret the word "projective structure" in a stricter sense, a projective structure in the previous sense + a choice of a spin bundle.
} 
Remark 1. Occasionally, it is also convenient to use, besides $\mu$ (the degree), another two parameters $\alpha=\mu-1$ (the order) and $v=\mu+1$ (the weight). Thus, in terms of $\alpha$,

$$
\mu=\alpha+1, \quad v=\alpha+2
$$

and, in terms of $v$,

$$
\alpha=v-2, \quad \mu=v-1 .
$$

The most natural parameter is perhaps the weight, because the weight is additive when forming tensor products. (In Berezin's theory of quantization (see e.g. [1], [8]) the inverse of the weight has the interpretation of "Planck's constant", that is, $h=$ $\frac{1}{v}=\frac{1}{\alpha+2}$.) The table below summarizes the two most important cases.

TABLE

\begin{tabular}{|l|c|c|c|}
\hline & order $\alpha$ & degree $\mu$ & weight $v$ \\
\hline Szegö & -1 & 0 & 1 \\
\hline Bergman & 0 & 1 & 2 \\
\hline
\end{tabular}

We can define an integral metric by requiring that

$$
(f, g)_{\mu} \stackrel{\text { deff }}{=} \int_{\Omega} L_{\mu}^{-1} f \bar{g} .
$$

Let us make this more precise, noting that the same definition makes sense also, more generally, for multiply connected planar domains (not only for connectivity two).

First of all, $f$ and $g$ are viewed as (holomorphic) sections of $\lambda^{\mu+1}$ (forms of degree $\frac{\mu+1}{2}$ or "differentials") so that, in particular, $F=L_{\mu}^{-1} f$ is a section of $\lambda^{1-\mu}$ (form of degree $\frac{1-\mu}{2}$ or "integral"). Then the integral of $F \bar{g}$, that is, of $F(z)(d z)^{-(\mu-1) / 2)} \cdot \overline{g(z)}(d \bar{z})^{(\mu+1) / 2}$, being a form of degree 1 , extended over any path $c$, is well-defined. It depends, however, if $\mu$ is odd, on orientation. Let $z=\varphi(t)$ be any parametrization of $c$. If $\mu$ is even ( $\alpha$ odd) then the integrand is

$$
F(z(t)) \overline{g(z(t))}\left(\overline{\frac{\varphi^{\prime}(t)}{\varphi^{\prime}(t)}}\right)^{\mu / 2} \underbrace{\varphi^{\prime}(t) \mid d t}_{|d z|}
$$

and if $\mu$ is odd ( $\alpha$ even)

$$
F(z(t)) \overline{g(z(t))}\left(\overline{\frac{\varphi^{\prime}(t)}{\varphi^{\prime}(t)}}\right)^{(\mu+1) / 2} \underbrace{\varphi^{\prime}(t) d t}_{d z}
$$

In the case at hand we have to integrate over the boundary $\partial \Omega$ which consists of two circles $\Gamma_{1}=\{z:|z|=1\}, \Gamma_{R}=\{z:|z|=R\}$. If $\mu$ is odd they have to be taken with opposite 
orientation. To begin with, let us consider any concentric circle $\Gamma_{r}=\{|z|=r\}(r>0)$ with the parametrization $z=\varphi(t)=r e^{i t}$ (that is, $\left.u=-t+i \log r\right)$. Then $\varphi^{\prime}(t)=i r e^{i t}, \varphi^{\prime}(t)=$ $-i r e^{-i t}$ so we find the integrand $F \bar{g} e^{-i \mu t} d t$ in both cases.

To proceed, we must give a meaning to $L_{\mu}^{-1}$. We begin by discussing the kernel of $L_{\mu}$. We observe that

$$
L_{\mu}\left(e^{i m u}\right)=U_{\mu}(m) e^{i m u}
$$

where we have put

$$
U_{\mu}(m)=\prod_{k=0}^{\alpha}(i m+\sqrt{-q}(\alpha-2 k)) .
$$

This is a polynomial of degree $\mu=\alpha+1$ in $m$ satisfying the symmetry condition

$$
U_{\mu}(-m)=(-1)^{\alpha} U_{\mu}(m) \text {. }
$$

This reflects the fact that $L_{\mu}$ intertwines with the actions of the automorphism group Aut $\Omega$ on "integrals". The latter group is generated by the following two types of transformations:

$1^{\circ}$ rotations about the origin $\left(z \mapsto z e^{i \theta}\right)$,

$2^{\circ}$ inversion about the circle $\Gamma_{\sqrt{R}}(z \mapsto R / z)$.

REMARK 2. If we employ the parameter $z$ instead of $u$ we have the canonical basis $\left\{z^{n}\right\}_{n \in \mathbb{Z}}$. Then $z^{n}(d z)^{-(\mu-1) / 2}$ corresponds to $e^{-i(n-(\mu-1) / 2)}(d u)^{-(\mu-1) / 2)} i^{(\mu-1) / 2}$ and $z^{n}(d z)^{(\mu+1) / 2}$ corresponds to $e^{-i(n+(\mu+1) / 2)}(d u)^{(\mu+1) / 2} i^{-(\mu+1) / 2}$. It follows that

$$
L_{\mu}\left(z^{n+\mu}\right)=Z_{\mu}(n) z^{n},
$$

where now

$$
Z_{\mu}(n)=\prod_{k=0}^{\alpha}\left(n+\frac{\alpha}{2}+i \sqrt{-q}(\alpha-2 k)\right)
$$

The symmetry condition now reads

$$
Z_{\mu}(-n)=(-1)^{\alpha} Z_{\mu}(n+\alpha+2) .
$$

From this we can in particular read off that the kernel (we are only interested in global solutions) is spanned by all functions $z^{n+\mu}$ such that $Z_{\mu}(n) \neq 0$. If $q<0$ (and below we will concentrate on that case) we see that $Z_{\mu}(n)=0$ iff $n=-\frac{\mu+1}{2}=-\frac{\alpha+2}{2}$. Then $L_{\mu}^{-1}$ is well-defined except when $n=-\frac{\mu+1}{2}$ :

$$
L_{\mu}^{-1} z^{n}=\frac{z^{n+\mu}}{Z_{\mu}(n)} \quad\left(n \neq-\frac{\mu+1}{2}\right)
$$


As $\mu$ is an integer, $n=-\frac{\mu+1}{2}$ can occur only if $\mu$ is odd, that is, $\alpha$ is even. We now find

$$
\begin{aligned}
\left(z^{n}, z^{n^{\prime}}\right)_{\mu} & =\delta_{n n^{\prime}} \int_{\partial \Omega} z^{n+\mu} \bar{z}^{n} / Z_{\mu}(n) \\
& =\delta_{n n^{\prime}}\left(\int_{\Gamma_{R}} R^{N+\mu} e^{i(n+\mu) t} R^{n} e^{-i n t} R e^{-i \mu t} d t+(-1)^{\mu} \int_{\Gamma_{1}} e^{i(n+\mu) t} e^{-i n t} e^{-i \mu t} d t\right) / Z_{\mu}(n) \\
& =\delta_{n n^{\prime}} \frac{R^{2 n+\mu+1}-(-1)^{\mu+1}}{Z_{\mu}(n)}=\delta_{n n^{\prime}} \frac{R^{2 n+\alpha+2}-(-1)^{\alpha+2}}{Z_{\mu}(n)} .
\end{aligned}
$$

As $Z_{\mu}(n)$ is real for all $n$ and positive for $n>-\frac{\mu+1}{2}$ we conclude from (1) that the metric is positive definite. In particular,

$$
\left\|z^{n}\right\|_{\mu}^{2}=\left(z^{n}, z^{n}\right)_{\mu}>0 .
$$

Finally, we complete the definition by putting (if $\mu$ is odd ( $\alpha$ even))

$$
\left\|z^{-(\mu+1) / 2}\right\|_{\mu}^{2}=\lim _{n \rightarrow-(\alpha+2) / 2} \frac{R^{2 n+\alpha+2}-1}{Z_{\mu}(n)}=\frac{2 \log R}{(\sqrt{-q})^{\alpha}(\alpha ! !)^{2}},
$$

where $\alpha ! !=\alpha .(\alpha-2) \ldots .4 .2$.

Every function $f$ can be uniquely written as $f=f_{+}+f_{-}+c z^{-(\mu+1) / 2}$ where $f_{+}$contains only terms $z^{n}$ with $n>-\frac{\mu+1}{2}$ and $f_{-}$contains only terms $z^{n}$ with $n<-\frac{\mu+1}{2}$ and $c$ is a constant. (Unless $\mu$ is odd ( $\alpha$ even), the last term is absent.) Thus

$$
f(d z)^{(\mu+1) / 2}=f_{+}(d z)^{(\mu+1) / 2}+f_{-}(d z)^{(\mu+1) / 2}+c\left(\frac{d z}{z}\right)^{(\mu+1) / 2} .
$$

Introduce (radial) measures $\mu_{+}$and $\mu_{-}$on $\Omega_{+}=\{0<|z|<R\}$ and $\Omega_{-}=\{1<|z|<\infty\}$ respectively, with densities

$$
w_{+}(z)=C(-1)^{\alpha} r^{\alpha} \sin ^{\alpha}(2 \sqrt{-q} \log r-2 \sqrt{-q} \log R)-(\text { if } r<1) C r^{\alpha} \sin ^{\alpha}(2 \sqrt{-q} \log r)
$$

and

$$
w_{-}(z)=C r^{\alpha} \sin ^{\alpha}(2 \sqrt{-q} \log r)-(\text { if } r>R)(-1)^{\alpha} C r^{\alpha} \sin ^{\alpha}(2 \sqrt{-q} \log r)
$$

respectively. $C$ is a constant which will be determined later (see (2)).

CLAIM. $\|f\|_{\mu}^{2}=\int_{\Omega_{+}}\left|f_{+}\right|^{2} d \mathcal{M}_{+}+\int_{\Omega_{-}}\left|f_{-}\right|^{2} d \mathcal{M}_{-}+|c|^{2}$.

EXAMPLE 5. If $2 \sqrt{-q} \cdot \log R=\pi$ then $\mu_{+}=\mu_{-}$(both measures are concentrated on $\Omega$ and coincide) with the density

$$
w(z)=C r^{\alpha} \sin ^{\alpha}\left(\frac{\pi \log r}{\log R}\right) .
$$

This is the only case when such a coincidence occurs. This, incidentally, disproves a previous conjecture made by us [10]. In what follows, we will refer to this case as the "weighted" case. 
Proof (of claim). It is sufficient to consider $\left\|z^{n}\right\|_{\mu}^{2}$. The case $n=-\frac{\mu+1}{2}$ is easy so consider e.g. the case $n=-\frac{\mu+1}{2}=-\frac{\alpha+2}{2}=-\frac{v}{2}$. We have

$$
\begin{aligned}
\int_{\Omega_{+}}|z|^{2 n} d M_{+}= & C \cdot\left(\int_{0}^{R} r^{2 n} \cdot r^{\alpha} \sin ^{\alpha}(2 \sqrt{-q} \log r-2 \sqrt{-q} \log R) r d r\right. \\
& \left.-(-1)^{\alpha} \int_{0}^{1} r^{2 n} \cdot r^{\alpha} \sin ^{\alpha}(2 \sqrt{-q} \log r) r d r\right) \cdot 2 \pi .
\end{aligned}
$$

Consider the last integral first. We have

$$
\sin ^{\alpha}(2 \sqrt{-q} \log r)=\left(\frac{e^{i 2 \sqrt{-q} \log n}-e^{-i 2 \sqrt{-q} \log n}}{2 i}\right)^{\alpha}=\left(\frac{1}{2 i}\right)^{\alpha} \sum_{k=0}^{\alpha}(-1)^{k}\left(\begin{array}{l}
\alpha \\
k
\end{array}\right) \underbrace{e^{i 2 \sqrt{-q} \log r(\alpha-2 k)}}_{r^{i 2 \sqrt{-q}(\alpha-2 k)}}
$$

so we find

$$
\begin{gathered}
C .2 \pi\left(\frac{1}{2 i}\right)^{\alpha} \sum_{k=0}^{\alpha}(-1)^{k}\left(\begin{array}{l}
\alpha \\
k
\end{array}\right) \int_{0}^{1} r^{2(n+(\alpha+2) / 2)+i 2 \sqrt{-q}(\alpha-2 k)} \frac{d r}{r} \\
=C \cdot \pi\left(\frac{1}{2 i}\right)^{\alpha} \sum_{k=0}^{\alpha}(-1)^{k}\left(\begin{array}{l}
\alpha \\
k
\end{array}\right) \frac{1}{n+\frac{\alpha+2}{2}+i 2 \sqrt{-q}(\alpha-2 k)} .
\end{gathered}
$$

Now we invoke the following polynomial identity.

Lemma.

$$
\frac{\alpha ! Y^{\alpha}}{\prod_{k=0}^{\alpha} X+k Y}=\sum_{k=0}^{\alpha}(-1)^{k}\left(\begin{array}{l}
\alpha \\
k
\end{array}\right) \frac{1}{X+k Y}
$$

Our integral becomes

$$
C . \pi\left(\frac{1}{2 i}\right)^{\alpha} \frac{\alpha !(-2 i \sqrt{-q})^{\alpha}}{\prod_{k=0}^{\alpha} n+\frac{\alpha+2}{2}+i \sqrt{-q}(\alpha-2 k)}=\frac{C \cdot \pi \cdot \alpha !(\sqrt{-q})^{\alpha}}{Z_{\mu}(n)}
$$

or simply $\frac{1}{Z_{\mu}(n)}$, if we fix the value of $C$ by

$$
C^{-1}=\pi \cdot \alpha \cdot(\sqrt{-q})^{\alpha} .
$$

In the same way we determine the first integral as $\frac{R^{2 n+\alpha+2}}{Z_{\mu}(n)}$.

Comparison with the previous formula for $\left\|z^{n}\right\|$ completes the proof in this case. The case $n<-\frac{\mu+1}{2}$ is similar. 
3. Boundedness of Hankel forms. Let us fix a projective structure on the annulus $\Omega=\Omega_{R}=\{z: 1<|z|<R\}$ and an integer $\mu$. We can then consider Hankel forms of the form

$$
\Gamma_{b}(f, g)=(f g, b)_{\mu}
$$

where $\mu^{\prime}+1=2(\mu+1)$ (or $\alpha^{\prime}+2=2(\alpha+2)$ or again $\left.v^{\prime}=2 v\right)$. The analytic function $b$ is its symbol. We ask when $\Gamma_{b}$ is bounded on the Hilbert space $A^{\alpha, 2}(\Omega)$ equipped with the metric $\|\cdot\|_{\mu}$ (all these norms are equivalent irrespective of $q<0$ ). As we can write down explicitly the reproducing kernel $K(z, w)$ in $A^{\alpha, 2}(\Omega)$ and work out its weak factorization (cf. [7] for details) a necessary condition is

$$
b(z)=O(K(z, z)) .
$$

To prove the converse we will use the representation of the metric given in Section 2, along with some simple facts about Besov space theory (see [9]).

Assume thus that (4) is fulfilled and let $f, g \in A^{\alpha, 2}(\Omega)$. We wish to prove that

$$
\left|\Gamma_{b}(f, g)\right| \leq C\|f\|_{\mu}\|g\|_{\mu}
$$

where $C$ depends on $b, q, \mu, R$. Set $h=f g$. Then by Section 2 (see the claim) we have formally

$$
\Gamma_{b}(f, g)=\int_{\Omega_{+}} \overline{b_{+}} h_{+} d \mathcal{M}_{+}+\int_{\Omega_{-}} \overline{b_{-}} h_{-} d M_{-}+\text {remainder }
$$

where $b=b_{+}+b_{-}, h=h_{+}+h_{-}$and the "remainder" involves only the Taylor coefficients with exponent $-\frac{\mu+1}{2}$. Let $\omega(z)=r \sin \left(\frac{\pi \log r}{\log R}\right) \approx$ Euclidean $\operatorname{dist}(z, \partial \Omega)$. Then (see [7])

$$
K(z, z) \approx \frac{1}{\omega(z)^{\alpha+2}}
$$

so that (4) is equivalent to

$$
b(z)=O\left(\frac{1}{\omega(z)^{\alpha+2}}\right) .
$$

Similarly by Schwarz's inequality,

$$
\int_{\Omega} \omega(z)^{\alpha+2}|h(z)| d x d y \leq C\|f\|_{\mu}\|g\|_{\mu}
$$

If we can prove that the functions $b_{+}$and $b_{-}$are subject to the estimate (6) and, similarly, that $h_{+}$and $h_{-}$satisfy (7), clearly (5) will follow.

To this end we have also to invoke the quasi-Banach spaces $A^{\alpha, p}=A^{\alpha, p}(\Omega)$ $(0<p \leqslant \infty, \alpha>-1)$, corresponding (if $\alpha+2>2 / p)$ to the quasi-norms

$$
\|f\|_{\alpha, p}=\left(\int_{\Omega}|f(z)|^{p} \omega(z)^{(\alpha+2) p / 2-2} d x d y\right)^{1 / p}
$$

if $\alpha+2 \leq 2 / p$ we must use instead a derivative of $f$ of sufficiently high order in the definition of the corresponding quasi-norms. Clearly, by what has been said, the norms 
$\|f\|_{\alpha, 2}$ and $\|f\|_{\mu}$ (for $\mu$ or $\alpha$ fixed) are equivalent, so that this definition agrees with the previous one if $p=2$. Our basic observation is now that a holomorphic function defined in $\Omega$ belongs to $A^{\alpha, p}(\Omega)$ iff its distributional boundary values (traces) on the circles $\Gamma_{R}$ and $\Gamma_{1}$ belong to the Besov spaces $B_{p}^{s, p}\left(\Gamma_{R}\right)$ and $B_{p}^{s, p}\left(\Gamma_{1}\right)$ respectively, where $s=\frac{1}{p}-$ $\frac{\alpha+2}{2}$. But the projections $f \mapsto f_{+}$and $f \mapsto f_{-}$are continuous in any Besov space $B_{p}^{s, q}\left(\Gamma_{r}\right)$. This concludes our argument.

Altogether we have now established the following theorem.

THEOREM. The Hankel form $\Gamma_{b}$ with symbol $b$, corresponding to some projective structure on the annulus $\Omega$, is bounded on the Hilbert space $A^{\alpha, 2}(\Omega)$ iff $b(z)=\mathrm{O}(K(z, z))$, where $K(z, w)$ is the reproducing kernel in $A^{\alpha, 2}(\Omega)$.

This generalizes the main result in [7] (the "weighted" case $2 \sqrt{-q} \cdot \log R=\pi$, see Example 5 in Section 2).

Remark 3. (Alternative approach) The boundedness of a Hankel form can also be proved along the following lines. We observe that if we have any integral metric which is rotation invariant, then the Hankel form $\Gamma_{b}$ with symbol $b$ formed with this metric formally can be written

$$
\Gamma_{b}(f, g)=\sum_{m, m \in \mathbb{R}} N(n+m) \overline{\hat{b}(n+m)} \hat{f}(n) \hat{g}(m),
$$

where $N(n)=\left\|z^{n}\right\|^{2}$ depends only on the metric. Therefore the symbols of one and the same Hankel form ${ }^{2}$ but corresponding to different projective structures differ from each other only by a multiplier transform. Therefore, the question of boundedness of a Hankel form corresponding to a given projective structure is, in principle, modulo a question of the boundedness of an appropriate multipler transform in Besov space, reduced to the "weighted" case.

4. Hankel forms in $S_{p}, 1<p<\infty$. The following theorem will be proved in this section.

THEOREM. For $1<p<\infty, \Gamma_{b}$ (considered as a form on $A^{\alpha, 2}(\Omega)$ ) is in the Schatten classes $S_{p}$ iff $b \in A^{\alpha^{\prime}, p}(\Omega)$, with $\alpha^{\prime}+2=2(\alpha+2)$.

First we consider the "weighted" case, i.e. $2 \sqrt{-q} \cdot \log R=\pi$. Then we have (up to a constant)

$$
\Gamma_{b}(f, g)=\int_{\Omega} \overline{b(z)} f(z) g(z) \omega^{\alpha^{\prime}}(z) d m(z)
$$

with $d m(z)=d x d y$. As in [6] (see especially Appendix 1), it readily follows that

$$
b \in A^{\alpha^{\prime}, p} \Rightarrow \Gamma_{b} \in S_{p}
$$

\footnotetext{
${ }^{2}$ Remember that from the abstract point of view (cf. [6]) the characteristic property of a Hankel form is that its value for given argument functions $f$ and $g$ depends only on the combination $f . g$.
} 
Indeed, in the case $p=1$ we can use the same argument as in the Proposition on p. 132 of [6], while the case $p=\infty$ is in Section 3 of the present paper. We note also that reproducing kernel of $A^{\alpha, 2}$ is given in [7], and we have

$$
K(z, z) \approx \omega(z)^{-(\alpha+2)}, \quad\left\|z^{n}\right\|_{\alpha} \approx \frac{\max \left(R^{2 n}, 1\right)}{|n|^{\alpha+1}} .
$$

The remaining cases $(1<p<\infty)$ are done by interpolation. For this and the proof of the converse we need the following auxiliary results.

LEMMA. Let $L(z, w)$ denote the reproducing kernel of $A^{\alpha^{\prime}, 2}$. If $s>-1, t>0$, and $t\left(\alpha^{\prime}+2\right)>s$, then

$$
\int_{\Omega}|L(z, \eta)|^{t} \omega(\eta)^{s} d m(\eta) \leq C \omega(z)^{s+2-t\left(\alpha^{\prime}+2\right)}
$$

As shown in [7], $e^{-i\left(\alpha^{\prime}+2\right)(u-\bar{v}) / 2} L\left(e^{-i u}, e^{-i \bar{v}}\right)$ is an elliptic function with poles of order $\alpha^{\prime}+2$ at the points of the lattice $G=2 \pi \mathbb{Z}+2(\log R) \mathbb{Z}$, so this lemma is an easy consequence of [11, Lemma 2.5].

Proposition. For $p>1$, consider the (Lebesgue) space $L^{\alpha^{\prime}, p}(\Omega)=\left\{f: \omega^{\alpha+2} f \in\right.$ $\left.L^{p}\left(\Omega, \omega^{-2} d m\right)\right\}$. Then there exists a projection $P: L^{\alpha^{\prime}, p}(\Omega) \rightarrow A^{\alpha^{\prime}, p}(\Omega)$ extending the orthogonal projection of $L^{\alpha^{\prime}, 2}(\Omega)$ onto $A^{\alpha^{\prime}, 2}(\Omega)$.

Proof. As shown in [6], the boundedness of the projection $P$

$$
P: L^{\alpha^{\prime}, p} \rightarrow A^{\alpha^{\prime}, p}, \quad P f(z)=\int_{\Omega} L(z, w) f(w) \omega(w)^{\alpha^{\prime}} d m(w),
$$

is the consequence of the following estimate,

$$
\int_{\Omega}|L(z, w)| \omega(w)^{\alpha} d m(w) \leq C \omega(z)^{-(\alpha+2)}
$$

In fact, for $p=\infty$, we see that if $f \in L^{\alpha^{\prime}, \infty}(\Omega)$, then

$$
|P f(z)| \leq\|f\|_{L^{\alpha, \infty}} \int_{\Omega}|L(z, w)| \omega(w)^{-(\alpha+2)} \omega(w)^{\alpha^{\prime}} d m(w) \leq C\|f\|_{L^{\alpha^{\prime}, \infty}} \omega(w)^{-(\alpha+2)} .
$$

So

$$
\|P f\|_{A^{\alpha^{\prime}, \infty}} \leq C\|f\|_{L^{\alpha^{\prime}, \infty}}
$$

The boundedness of $P$ in the case $p=1$ can be proved similarly. By interpolation, it is true for $1<p<\infty$. However, the inequality (8) follows then from the Lemma. This proves the proposition.

Corollary. For $p>1, \frac{1}{p}+\frac{1}{q}=1$, we have $\left(A^{\alpha^{\prime}, p}\right)^{*}=A^{\alpha^{\prime}, q}$, with the pairing

$$
\langle f, g\rangle=\int_{\Omega} f(z) \overline{g(z)} \omega(z)^{\alpha^{\prime}} d m(z) \text {. }
$$


REMARK. Of course, this corollary could also have been proved using the theory of Besov spaces (cf. Section 3).

It follows from [6] that

$$
\begin{aligned}
\operatorname{tr}\left(\Gamma_{b} \Gamma_{c}^{*}\right) & =\int_{\Omega} \int_{\Omega} K^{2}(z, \eta) b(\eta) \overline{c(z)} \omega(z)^{\alpha^{\prime}} \omega(\eta)^{\alpha^{\prime}} d m(z) d m(\eta) \\
& =\int_{\Omega}(\Phi b)(z) \overline{c(z)} \omega(z)^{\alpha^{\prime}} d m(z),
\end{aligned}
$$

where

$$
(\mathscr{g} b)(z)=\int_{\Omega} K^{2}(z, \eta) b(\eta) \omega(\eta)^{\alpha^{\prime}} d m(\eta) .
$$

By the Corollary, we see that our theorem will follow from the claim that

$$
\mathscr{I}: A^{\alpha^{\prime}, p}(\Omega) \rightarrow A^{\alpha^{\prime}, p}(\Omega)
$$

is invertible. The operator $\mathscr{I}$ is a convolution in the sense that

$$
\mathscr{I} f(z)=\sum_{n=-\infty}^{\infty} \frac{N_{n}}{\tilde{M}_{n}} \hat{f}_{n} z^{n}, \quad f(z)=\sum_{n=-\infty}^{\infty} \hat{f}_{n} z^{n},
$$

where $\frac{1}{\bar{M}_{n}}=\sum_{k+l=n} \frac{1}{M_{k} M_{l}}, M_{l}=\left\|z^{\prime}\right\|_{\alpha}^{2}, N_{l}=\left\|z^{\prime}\right\|_{\alpha^{\prime}}^{2}$. So

$$
\mathscr{I}^{-1} f(z)=\sum_{n} \frac{\tilde{M}_{n}}{N_{n}} \hat{f}_{n} z^{n}, \quad f(z)=\sum_{n} \hat{f}_{n} z^{n}
$$

which can then be expressed as

$$
g^{-1} f(z)=\int_{\Omega} G(z, \eta) f(\eta) \omega(\eta)^{\alpha^{\prime}} d m(\eta),
$$

with $G(z, \eta)=\sum_{n} \frac{\bar{M}_{n}}{N_{n}^{2}} z^{n} \bar{\eta}^{n}$. By [6], the boundedness of $\mathscr{I}^{-1}$ will follow from

$$
\int_{\Omega}|G(z, \eta)| \omega(\eta)^{\alpha} d m(\eta) \leq C \omega(z)^{-(\alpha+2)}
$$

For $n>0$, it is shown in [7, Theorem 2, Section 5] that

$$
\begin{gathered}
\frac{1}{\bar{M}_{n}}=\sum_{k=0}^{n} \frac{1}{M_{k} M_{n-k}}+O\left(\frac{n^{\alpha+1}}{R^{2 n}}\right), \\
\frac{1}{M_{n}}=C \frac{\prod_{k=0}^{n}(\log R(2 n+\alpha+2)+i \pi(\alpha-2 k))}{R^{2 n+\alpha+2}+(-1)^{\alpha+1}} .
\end{gathered}
$$

It is easy to see that

$$
\frac{1}{M_{n}}=C \frac{n^{\alpha+1}}{R^{2 n}}+O\left(\frac{n^{\alpha}}{R^{2 n}}\right)
$$


So

$$
\frac{1}{\bar{M}_{n}}=C \sum_{k=0}^{n}\left(\frac{k^{\alpha+1}}{R^{2 k}}+O\left(\frac{k^{\alpha}}{R^{2 k}}\right)\right)\left(\frac{(n-k)^{\alpha+1}}{R^{2(n-k)}}+O\left(\frac{(n-k)^{\alpha}}{R^{2(n-k)}}\right)\right)+O\left(\frac{n^{\alpha+1}}{R^{2 n}}\right) .
$$

We also see that if $t, s>0$, then

So

$$
\begin{aligned}
\sum_{k=0}^{n} k^{s}(n-k)^{t} & =n^{t+s+1} \sum_{k=0}^{n}\left(\frac{k}{n}\right)^{t}\left(1-\frac{k}{n}\right)^{s} \frac{1}{n} \\
& =n^{t+s+1}\left(\int_{0}^{1} x^{t}(1-x)^{s} d x+O\left(\frac{1}{n}\right)\right) \\
& =C n^{t+s+1}+O\left(n^{t+s}\right) .
\end{aligned}
$$

As

$$
\frac{1}{\tilde{M}_{n}}=C \frac{n^{2 \alpha+3}}{R^{2 n}}+O\left(\frac{n^{2 \alpha+2}}{R^{2 n}}\right) .
$$

$$
N_{n}=\left\|z^{n}\right\|_{\alpha^{\prime}}^{2}=C \frac{R^{2 n}}{n^{2 \alpha+3}}+O\left(\frac{R^{2 n}}{n^{2 \alpha+2}}\right)
$$

we get

$$
\frac{N_{n}}{\tilde{M}_{n}}=C+O\left(\frac{1}{n}\right), \quad n>0 .
$$

Similarly, we can prove the same estimate for $n<0$ (with the same constant $C$, but this not essential for our proof below). Therefore

$$
\begin{aligned}
G(z, n) & =\sum_{n=-\infty}^{\infty} \frac{\tilde{M}_{n}}{N_{n}^{2}}(z \bar{\eta})^{n}=\sum_{n=-\infty}^{\infty} \frac{\tilde{M}_{n}^{2}}{N_{n}^{2}} \frac{1}{\tilde{M}_{n}}(z \bar{\eta})^{n} \\
& =C \sum_{n=\infty}^{\infty} \frac{1}{\tilde{M}_{n}}(z \bar{\eta})^{n}+\sum_{n=-\infty}^{\infty} \frac{C_{n}}{\tilde{M}_{n}}(z \bar{\eta})^{n} \\
& =G_{1}+G_{2},
\end{aligned}
$$

with $\left|C_{n}\right| \leq C \frac{1}{|n|}$.

Notice that $G_{1}=K^{2}(z, \eta)$, so we have

$$
\int_{\Omega}\left|G_{1}(z, \eta)\right| \omega(\eta)^{\alpha} d m(\eta)=K(z, z) \leq C \omega(z)^{-(\alpha+2)}
$$

For $G_{2}$, we have

$$
\begin{aligned}
\int_{\Omega}\left|G_{2}(z, \eta)\right| \omega(\eta)^{\alpha} d m(\eta) & \leq \sum_{n=-\infty}^{\infty} \frac{\left|C_{n}\right|}{\tilde{M}_{n}} \int_{\Omega}|z \bar{\eta}|^{n} \omega(\eta)^{\alpha} d m(\eta) \\
& =\sum_{n \text { even }}+\sum_{n \text { odd }}=\sum^{\prime}+\sum^{\prime \prime}
\end{aligned}
$$


and

$$
\begin{aligned}
\sum^{\prime} & =\sum_{n=-\infty}^{\infty} \frac{\left|C_{2 n}\right|}{\tilde{M}_{2 n}}|z|^{2 n} \int_{\Omega}|\bar{\eta}|^{2 n} \omega(\eta)^{\alpha} d m(\eta)=\sum_{n=-\infty}^{\infty} \frac{\left|C_{2 n}\right|}{\tilde{M}_{2 n}}\left|z^{2 n}\right| M_{n} \\
& \leq C \frac{1}{n} \frac{n^{2 \alpha+3}}{\max \left(R^{4 n}, 1\right)} \frac{\max \left(R^{2 n}, 1\right)}{n^{\alpha+1}}=C \sum_{n=-\infty}^{\infty} n^{\alpha+1} \max \left(R^{2 n}, 1\right) \\
& \leq C \max \left(\left(1-\frac{1}{|z|^{2}}\right)^{-(\alpha+2)},\left(1-\frac{|z|^{2}}{R^{2}}\right)^{-(\alpha+2)}\right) \\
& \leqslant C \omega(z)^{-(\alpha+2)} .
\end{aligned}
$$

Since $|z|^{2 n+1} \leq C|z|^{2 n}$, the second term $\Sigma^{\prime \prime}$ can easily be seen to be dominated by the first one. So eventually we get the estimate (2). This proves the Theorem in the "weighted case".

In the general case, $q<0$, we see that $\Gamma_{b}=\Gamma_{b_{1}}^{\prime}$, where $\Gamma^{\prime}$ is the Hankel form in the "weighted" case, and $b_{1}$ is a convolution of $b$. In fact;

$$
b_{1}=\sum_{n} \frac{N_{n}}{N_{n}^{\prime}} \hat{b}_{n} z^{n}, \quad b=\sum_{n} \hat{b}_{n} z^{n},
$$

where $N_{n}$, as before, is the norm of $z^{n}$ and $N_{n}^{\prime}$ is the corresponding quantity in the weighted case. Using the above technique, we can prove that $b \in A^{\alpha^{\prime}, p}$ iff $b_{1} \in A^{\alpha^{\prime}, p}$. We omit the details. So this proves the main theorem of this Section.

5. Decomposition theorems. Let $d(\xi, \eta)$ be the Poincaré distance on $\Omega$. We decompose now $\Omega$ in the following way: With $R_{1 / 2}=\frac{1+R}{2}$, let

$$
\begin{gathered}
\Omega=\bigcup_{m, j} \Omega_{j}^{m}, \\
\Omega_{j}^{m}=\left\{z=r e^{i \theta}: 1+\frac{R_{1 / 2}-1}{2^{m+1}}<r<1+\frac{R_{1 / 2}-1}{2^{m-1}}, \frac{j-1}{2^{m}} 2 \pi<\theta<\frac{j+1}{2^{m}} 2 \pi\right\}, \\
m=1,2, \ldots, j=1,2, \ldots, 2 m-1 . \\
\Omega_{j}^{m}=\left\{z=r e^{i \theta}: R-\left(R-R_{1 / 2}\right) 2^{m-1}<R-\left(R_{1 / 2}-R\right) 2^{m+1}, 2^{m}(j-1) 2 \pi<\theta<2^{m}(j+1) 2 \pi\right\}, \\
m=-1,-2, \ldots, j=1,2, \ldots, 2^{-m} .
\end{gathered}
$$

Let $\omega_{j}^{m}$ be the centers of $\Omega_{j}^{m}$, and $D_{j}^{m}$ the corresponding "squares" with centers $\omega_{j}^{m}$, contained in $\Omega_{j}^{m}$ and disjointly decomposing $\Omega$. Then we see that it is a 1-lattice. We can find an $\varepsilon$-lattice similarly.

THEOREM. Let $\frac{1}{\alpha+2}<p<\infty$ and $\varepsilon>0$. Let $\left\{\eta_{j}\right\}$ be the $\varepsilon$-lattice described above. Then for sufficiently small $\varepsilon$, every function $f \in A^{\alpha^{\prime}, p}(\Omega)$ can be represented as

$$
f(z)=\sum_{i} \lambda_{i} L\left(z, \eta_{i}\right) \omega\left(\eta_{i}\right)^{\alpha+2}
$$


with $\|f\|_{A^{\alpha^{\prime}, p}} \approx \inf \sum_{i}\left|\lambda_{i}\right|^{p}$, where the infimum is taken over the all the families $\lambda_{i}$ arising in this way.

The proof of the theorem is essentially the same as that of Theorem 2.2 in [11]. Here we only prove the case $\frac{1}{\alpha+2}<p<1$.

Let $\left\{\eta_{i}\right\}$ be the $\varepsilon$-lattice, and $D_{i}$ and $\Omega_{i}$ be some enumerations of $D_{i}^{m}$ and $\Omega_{i}^{m}$, respectively. Define the operators $S$ and $T$ by the following:

$$
\begin{gathered}
S: f \mapsto\left\{\omega\left(\eta_{i}\right)^{-(\alpha+2)} \int_{D_{i}} f(\eta) \omega(\eta)^{p} d m(\eta)\right\}, \quad f \in A^{\alpha^{\prime}, p}(\Omega), \\
T:\left\{\lambda_{i}\right\} \mapsto \sum_{i} \lambda_{i} L_{\alpha^{\prime}}\left(z, \eta_{i}\right) \omega\left(\eta_{i}\right)^{\alpha+2}, \quad\left\{\lambda_{i}\right\} \in l^{p} .
\end{gathered}
$$

Then, using Lemma 1 and subharmonicity, it is easy to prove that $T$ is bounded from $l^{p}$ to $A^{\alpha^{\prime}, p}$, and that $S$ is bounded from $A^{\alpha^{\prime}, p}$ to $l^{p}$. Now

$$
\begin{aligned}
(1-T S) f(z) & =\int_{\Omega} L(z, \eta) f(\eta) \omega(\eta)^{\alpha^{\prime}} d m(\eta)-\sum_{i} \int_{D_{i}} f(\eta) \omega(\eta)^{\alpha^{\prime}} d m(\eta) L\left(z, \eta_{i}\right) \\
& =\sum_{i} \int_{D_{i}} L(z, \eta) f(\eta) \omega(\eta)^{\alpha^{\prime}} d m(\eta)-\sum_{i} \int_{D_{i}} f(\eta)^{\alpha^{\prime}} L\left(z, \eta_{i}\right) d m(\eta) \\
& =\sum_{i} \int_{D_{i}} f(\eta)\left[L(z, \eta)-L\left(z, \eta_{i}\right)\right] \omega(\eta)^{\alpha^{\prime}} d m(\eta) .
\end{aligned}
$$

Notice that $\omega(\eta) \approx \omega\left(\eta_{i}\right)$ when $\eta \in \Omega_{i}$, so, by subharmonicity, we have

$$
\begin{aligned}
\|(1-S T) f\|_{A^{\alpha, p}}^{p} \leq & \sum_{i} \int_{\Omega} \omega(z)^{p(\alpha+2)-2} d m(z)\left|\int_{D_{i}} f(\eta)\left[L(z, \eta)-L\left(z, \eta_{i}\right)\right] \omega(\eta)^{\alpha^{\prime}} d m(\eta)\right|^{p} \\
\leq & C \sum_{i} \int_{\Omega} \omega(z)^{p(\alpha+2)-2} d m(z) \\
& \times \int_{\Omega_{i}}\left|f(\eta)\left[L(z, \eta)-L\left(z, \eta_{i}\right)\right] \omega(\eta)^{\alpha^{\prime}+2}\right|^{p} \omega(\eta)^{-2} d m(\eta) \\
\leq & C \sum_{i} \int_{\Omega_{i}}|f(\eta)|^{p} \omega(\eta)^{\left(\alpha^{\prime}+2\right) p} \omega(\eta)^{-2} d m(\eta) \\
& \times \int_{\Omega}\left|L(z, \eta)-L\left(z, \eta_{i}\right)\right| \omega(\eta)^{p(\alpha+2)-2} d m(\eta) .
\end{aligned}
$$

As $L(z, w)=L\left(e^{-i u}, e^{-i v}\right)=e^{i(\alpha+2)(u-\bar{v}) / 2} e(u-\bar{v})$, where $e$ is an elliptic function of order $\alpha^{\prime}+2$ with poles in the lattice $G=2 \pi \mathbb{Z}+i 2 \Lambda \mathbb{Z}$, so using Lemma 1 we see that if $\eta \in \Omega_{i}$, then

$$
\int_{\Omega}\left|L(z, \eta)-L\left(z, \eta_{i}\right)\right|^{p} \omega(z)^{p(\alpha+2)-2} d m(z) \leq C \varepsilon^{p} \omega(\eta)^{-p(\alpha+2)} .
$$


Then, because $\left\{\Omega_{i}\right\}$ intersect at most finitely many times (the maximum number is independent of $\varepsilon$ ), we get

$$
\begin{aligned}
\|(1-S T) f\|_{A^{\alpha, p}}^{p} & \leq C \varepsilon^{p} \sum_{i} \int_{\Omega_{i}}|f(\eta)|^{p} \omega(\eta)^{p(\alpha+2)-2} d m(\eta) \\
& \leq C \epsilon^{p} \int_{\Omega}|f(\eta)|^{p} \omega^{p(\alpha+2)-2}(\eta) d m(\eta) \\
& \leq C \varepsilon^{p}\|f\|_{A^{\alpha^{\prime}, p}}^{p}
\end{aligned}
$$

If we choose $\varepsilon$ sufficient small, then we have $\|1-S T\|<1$. So $S$ is onto, which proves the theorem.

6. Hankel forms in $S_{p}, 0<p<1$. With the help of the result in the last section, we can give $S_{p}$-results for Hankel forms for $\frac{1}{\alpha+2}<p<1$.

THEOREM. For $\frac{1}{\alpha+2}<p<1$, the Hankel form $\Gamma_{b}$ on $A^{\alpha, 2}(\Omega)$ is in $S_{p}$ iff $b \in A^{\alpha^{\prime}, p}$.

Proof. First we consider the "weighted" case, i.e. $2 \sqrt{-q} \log R=\pi$. Then we have

$$
\Gamma_{b}(f, g)=\int_{\Omega} \overline{b(z)} f(z) g(z) \omega(z)^{\alpha^{\prime}} d m(z)
$$

with $\alpha^{\prime}=2 \alpha+2$. If $b \in A^{\alpha^{\prime}, p}$, then, by Theorem 3 ,

$$
b(z)=\sum_{i} \lambda_{i} L\left(z, \eta_{i}\right) \omega\left(\eta_{i}\right)^{\alpha+2}
$$

with $\sum_{i}\left|\lambda_{i}\right|^{p}<\infty$. So

$$
\Gamma_{b}=\sum_{i} \lambda_{i} f_{i} \otimes f_{i}
$$

where $f_{i}(z)=K\left(z, \eta_{i}\right) \omega\left(\eta_{i}\right)^{(\alpha+2) / 2}$. Then it follows that $\left\|\Gamma_{b}\right\|_{S_{p}}^{p} \leq \sum_{i}\left|\lambda_{i}\right|^{p}<\infty$.

Now suppose, conversely, that $\Gamma_{b} \in S_{p}$. Writing $b=b_{+}+b_{-}$, we will prove that $b_{+}, b_{-} \in A^{\alpha^{\prime}, p}(\Omega)$. The proof of $b_{-} \in A^{\alpha^{\prime}, p}$ will be the same as that for $b_{+}$. So we assume $b_{-}=0$. For any $f \in A^{\alpha, 2}, f_{-}=0$, we can extend it naturally to an analytic function on $D_{R}=\{z:|z|<R\}$, and denote it also by $f$, which is in $A^{\alpha, 2}\left(D_{R}\right)$. Let $H_{b}$ denote the Hankel form on $A^{\alpha, 2}\left(D_{R}\right)$ with the weight $\alpha^{\prime}+2$, i.e.

$$
H_{b}(f, g)=\int_{D_{R}} \overline{b(z)} f(z) g(z)\left(1-\frac{|z|^{2}}{R^{2}}\right)^{\alpha^{\prime}} d m(z) .
$$

Then direct calculation shows that

$$
\Gamma_{b}(f, g)=H_{b_{1}}(f, g)
$$

with

$$
b(z)=\sum_{n=0}^{\infty} C_{n} \hat{b}_{1}(n) z^{n}, \quad C_{n}=C+\frac{C_{1}}{n}+\frac{C_{2}}{n^{2}}+\ldots
$$


From $\Gamma_{b} \in S_{p}$, we see that $H_{b_{1}} \in S_{p}$, and then $b_{1} \in A^{\alpha^{\prime}, p}\left(D_{R}\right) \subset A^{\alpha^{\prime}, p}(\Omega)$ (see e.g. [5]). On the other hand, as in Section 4 we have

$$
b(z)=\int_{D_{R}} b_{1}(w) G(z, w)\left(1-\frac{|w|^{2}}{R^{2}}\right)^{\alpha^{\prime}} d m(w),
$$

with $G(z, w)=\sum_{n=0}^{\infty} C_{n} \frac{(z \bar{w})^{n}}{N_{n}}$, and a (different) constant $C_{n}=C+\frac{C_{1}}{n}+\frac{C_{n}}{n^{2}}+\cdots$. The same method as in Section 4 will give us

$$
G(z, w)=C\left(1-\frac{(z \bar{w})}{R^{2}}\right)^{-\alpha^{\prime}-2}+L_{1}
$$

Then

$$
b=C b_{1}+S b_{1}, \quad S b_{1}(z)=\int_{D_{R}} b_{1}(w) L_{1}(z, w)\left(1-\frac{|w|^{2}}{R^{2}}\right)^{\alpha^{\prime}} d m(w) .
$$

As $L_{1}$ has lower singularity than that of $\left(1-\frac{z \bar{w}}{R^{2}}\right)^{-\alpha^{\prime}-2}$, it is not difficult to prove that

$$
S: A^{\alpha^{\prime}, p}\left(D_{R}\right) \rightarrow A^{\alpha^{\prime}, p}\left(D_{R}\right) .
$$

So we get $b \in A^{\alpha^{\prime}, p}\left(D_{R}\right) \subset A^{\alpha^{\prime}, p}(\Omega)$. This finishes the proof of the theorem.

In the general case $q<0$, as noted in Section 3, the reproducing kernel of $A^{\alpha^{\prime}, 2}$ (with respect to the integral metric) can also be decomposed similarly. Then the same method as the above will work. So we have proved our theorem.

7. The case $\alpha$ non-integer. Hitherto we have assumed that the parameter $\alpha$ (or, equivalently, $\mu$ or $v$ ) is an integer. Now we drop this assumption, assuming only that $\alpha>-1$. Then we must restrict ourselves to the "weighted" case, because we do not know how to define the metric otherwise. Thus, from now on $A^{\alpha, 2}(\Omega)$ denotes the space of all analytic functions $f$ defined over $\Omega$ such that

$$
\|f\|_{\alpha}^{2}=\int_{\Omega}|f(z)|^{2} r^{\alpha} \sin ^{\alpha}\left(\frac{\pi \log r}{\log R}\right) d x d y<\infty,
$$

where $z=x+i y, r=|z|$. integral

To determine the reproducing kernel $K_{\alpha}(z, w)$ in $A^{\alpha, 2}(\Omega)$ we must compute the

$$
\left\|z^{n}\right\|_{\alpha}^{2}=\int_{1}^{R} r^{2 n} r^{\alpha} \sin ^{\alpha}\left(\frac{\pi \log r}{\log R}\right) r d r .2 \pi
$$

for $n \in \mathbb{Z}$.

We notice that $\sin ^{\alpha}\left(\frac{\pi \log r}{\log R}\right)=\left(\frac{e^{i(\pi \log r \log R)}-e^{-i(\pi \log r \log R)}}{2 i}\right)^{\alpha}=\left(\frac{i}{2}\right)^{\alpha} e^{-i \alpha(\pi \log r \log R)}\left(1-e^{-i 2(\pi \log r \log R)}\right)^{\alpha}$. 
Introduce the new variable of integration

$$
\zeta=e^{i(2 \pi \log r / \log R)}=r^{2 \pi i / \log R}
$$

so that

$$
\frac{d \zeta}{\zeta}=\frac{2 \pi i}{\log R} \cdot \frac{d r}{r}
$$

Then our integral can be written

$$
\left\|z^{n}\right\|_{\alpha}^{2}=\frac{\log R}{2^{\alpha} i^{\alpha+1}} \int_{|\zeta|=1} \zeta^{\log R(n+(\alpha+2) / 2) / \pi i-(\alpha / 2)}(1-\zeta)^{\alpha} \frac{d \zeta}{\zeta}
$$

where we integrate over the unit circumference counterclockwise starting at the point $\zeta=1$.

Consider, quite generally, the integral

$$
J=\int_{|\zeta|=1} \zeta^{\beta}(1-\zeta)^{\alpha} \frac{d \zeta}{\zeta}
$$

where $\alpha, \beta$ are complex numbers with $\operatorname{Re} \alpha>-1,(1-\xi)^{a}$ is a branch of that function defined in $\mathbb{C}$ cut along the interval $(1, \infty)$ which takes real values on $(-1, \infty), \zeta^{\beta}$ is a branch defined in $\mathbb{C}$ cut along $(0, \infty)$ which has a real limit when $\zeta$ approaches a point on $(0, \infty)$ through values with positive imaginary part (i.e. $\lim _{\substack{\xi \rightarrow \xi \\ \operatorname{lm} \zeta>0}} \xi^{\beta}=\xi^{\beta}$ whenever $\left.\xi>0\right)$ and, finally, we integrate along any "contour" in $\mathbb{C}$ cut along $(0, \infty)$, connecting 1 with itself, starting with values of $\zeta$ with positive imaginary part and ending with values with negative imaginary part (e.g. the unit circumference taken counterclockwise).

FACT. $J=\left(e^{2 \pi i \beta}-1\right) B(\beta, \alpha+1)$ where $B$ is the Euler beta function.

Note. $J=0$ if $\beta=1,2,3, \ldots$

In our case we have $\beta=\frac{\log R}{\pi i}\left(n+\frac{\alpha+2}{2}\right)-\frac{\alpha}{2}$. As, quite generally,

$$
B(\beta, \alpha+1)=\frac{\Gamma(\beta) \Gamma(\alpha+1)}{\Gamma(\beta+\alpha+1)},
$$

where $\Gamma$ is the Euler gamma function, we obtain

$$
\left\|z^{n}\right\|_{\alpha}^{2}=e^{-i \alpha \pi} \cdot \frac{\log R}{2^{\alpha} i^{\alpha+1}} \cdot \frac{\Gamma(\alpha+1) \Gamma\left(-\frac{\alpha}{2}+\frac{\log R}{\pi i}\left(n+\frac{\alpha+2}{2}\right)\right)\left(R^{2 n+\alpha+2}-e^{i \alpha \pi}\right)}{\Gamma\left(\frac{\alpha}{2}+\frac{\log R}{\pi i}\left(n+\frac{\alpha+2}{2}\right)+1\right)} .
$$

In particular, it follows from Stirling's formula that

$$
\left\|z^{n}\right\|_{\alpha}^{2} \sim \frac{1}{n^{\alpha+1}}
$$


For $\alpha=0,1,2, \ldots$ this agrees with what was done in [7]. As

$$
K_{\alpha}(z, w)=\sum_{n=-\infty}^{\infty} \frac{(z \bar{w})^{n}}{\left\|z^{n}\right\|_{\alpha}^{2}}
$$

we can now, in principle, write down a "closed" expression for the reproducing kernel. From it one can read off that it has a singularity of the type $(1-z \bar{w})^{-(\alpha+2)}$, as expected.

The same method as in Section 4 will give the $S_{p}$-results for the Hankel forms, $1<p<\infty$. Furthermore we have the following weak factorization.

THEOREM. There exist finitely many functions $u_{i}$ and $v_{i}$ such that

and

$$
L(z, w)=\sum_{i} u_{i}(z, w) v_{i}(z, w)
$$

$$
\sum_{i}\left\|u_{i w}\right\|_{\alpha}\left\|v_{i w}\right\|_{\alpha} \leq C \omega(w)^{-(\alpha+2)}
$$

where $u_{i w}(z)=u_{i}(z, w), v_{i w}(z)=v_{i}(z, w)$.

We will prove the theorem by using the methods in Section 4.

Write $L=L_{+}+L_{-}, K=K_{+}+K_{-}$, with $K_{+}=\sum_{n=0}^{\infty} \frac{(z \bar{w})^{n}}{M_{n}}, K_{-}=\sum_{n=-1}^{\infty} \frac{(z \bar{w})^{n}}{M_{n}}$, and the same for $L$. By Stirling's formula,

$$
\frac{\Gamma\left(-\frac{\alpha}{2}+\frac{\log R}{\pi i}\left(n+\frac{\alpha+2}{2}\right)\right)}{\Gamma\left(\frac{\alpha}{2}+\frac{\log R}{\pi i}\left(n+\frac{\alpha+2}{2}\right)+1\right)}=C n^{-\alpha-1}\left(1+\frac{C_{1}}{n}+\frac{C_{2}}{n^{2}}+\ldots\right)
$$

where $C_{i}$ are constants. So we get

$$
\left\|z^{n}\right\|_{\alpha}=C\left(R^{2 n+\alpha+2}-e^{i \alpha \pi}\right)|n|^{-\alpha-1}\left(1+\frac{C_{1}}{n}+\frac{C_{2}}{n^{2}}+\cdots\right)
$$

For $n \geq 0$, as $\frac{n^{l}}{R^{2 n}} \rightarrow 0$, for any $l$, we have for a positive $m$, say $m \geq \frac{3 \alpha+8}{2}$,

$$
M_{n}=C R^{2 n} n^{-\alpha-1}\left(1+\frac{C_{1}}{n}+\frac{C_{2}}{n^{2}}+\ldots+\frac{C_{m-1}}{n^{m-1}}+O\left(\frac{1}{n^{m}}\right)\right)
$$

Define $u_{1}(z, w)=\sum_{n=0}^{\infty} \frac{n^{\alpha+1}}{R^{2 n}}(z \bar{w})^{n}$. Then the same calculation as in Section 4 gives us

$$
u_{1}(z, w)=C K_{+}(z, w)+r_{1}(z, w) \quad \text { with } \quad r_{1}(z, w)=\sum_{n=0}^{\infty} C_{n} \frac{n^{\alpha}}{R^{2 n}}(z \bar{w})^{n},\left|C_{n}\right| \leq C,
$$


and

$$
\int_{\Omega}\left|u_{1}(z, w)\right|^{2} \omega(z)^{\alpha} d m(z) \leq C \omega(w)^{-(\alpha+2)}
$$

Writing $u_{1}^{2}(z, w)=\sum_{n=0}^{\infty} \frac{(z \bar{w})^{n}}{\tilde{M}_{n}}$, we then have (see Section 4)

$$
\frac{1}{\tilde{M}_{n}}=\frac{n^{2 \alpha+3}}{R^{2 n}}\left(1+\frac{C_{1}}{n}+\ldots+O\left(\frac{1}{n^{m}}\right)\right), \quad \frac{N_{n}}{\tilde{M}_{n}}=C+\frac{C_{1}}{n}+\ldots+O\left(\frac{1}{n^{m}}\right),
$$

where as before $N_{n}=\left\|z^{n}\right\|_{\alpha^{\prime}}^{2}$. So

$$
\begin{aligned}
L_{+}(z, w) & =\sum_{n=0}^{\infty} \frac{1}{N_{n}}(z \bar{w})^{n}=\sum_{n=0}^{\infty} \frac{\tilde{M}_{n}}{N_{n}} \frac{1}{\bar{M}_{n}}(z \bar{w})^{n}=C \sum_{n=0}^{\infty}\left(\frac{1}{\tilde{M}_{n}}+\frac{1}{N_{n}^{(1)}}\right)(z \bar{w})^{n} \\
& =C u_{1}^{2}(z, w)+L_{+}^{(1)}(z, w),
\end{aligned}
$$

with

$$
\frac{1}{N_{n}^{(1)}}=\frac{n^{2 \alpha+2}}{R^{2 n}}\left(1+\frac{C_{1}}{n}+\ldots+\frac{C_{m-2}}{n^{m-2}}+O\left(\frac{1}{n^{m-1}}\right)\right) .
$$

Define $u_{2}(z, w)=\sum_{n=0}^{\infty} \frac{n^{\alpha}}{R^{2 n}}(z \bar{w})^{n}$. Then essentially the same arguments will imply

with

$$
L_{+}^{(1)}=C u_{2} u_{1}+L_{+}^{(2)}
$$

$$
\begin{gathered}
L_{+}^{(2)}=C \sum_{n=0}^{\infty} \frac{1}{N_{n}^{(2)}}(z \bar{w})^{n}, \quad\left\|u_{2 w}\right\|_{\alpha}^{2} \leq C \omega(w)^{-(\alpha+2)}, \\
\frac{1}{N_{n}^{(2)}}=\frac{n^{2 \alpha+1}}{R^{2 n}}\left(1+\frac{C_{1}}{n}+\ldots+\frac{C_{m-3}}{n^{m-3}}+O\left(\frac{1}{n^{m-2}}\right)\right) .
\end{gathered}
$$

Continuing this process, until the $s$-th step, with $s \geq \frac{3 \alpha+6}{2}$, we get (with different $u_{i}$ )

$$
L_{+}=u_{1} v_{1}+\ldots+u_{s-1} v_{s-1}+L_{+}^{(s)}
$$

and

$$
L_{+}^{(s)}(z, w)=\sum_{n=0}^{\infty} C_{n} \frac{n^{2 \alpha+3-s}}{R^{2 n}}(z \bar{w})^{n}, \quad C_{n}=C+O\left(\frac{1}{n}\right) .
$$

Let $u_{s}(z, w)=L_{+}^{(s)}(z, w), v_{s}=1$. Then we see that

$$
u_{s}^{2}(z, w)=\sum_{n=0}^{\infty} q_{n}(z \bar{w})^{n}, \quad\left|q_{n}\right| \leq C \frac{n^{2(2 \alpha+3-s)+1}}{R^{2 n}} .
$$

Therefore

$$
\begin{aligned}
\left\|u_{s w}\right\|_{\alpha}^{2} & =\int_{\Omega}\left|u_{s}(z, w)\right|^{2} \omega(z)^{\alpha} d m(z) \\
& =\sum_{n \text { odd }}+\sum_{n \text { even }}=\Sigma^{\prime}+\Sigma^{\prime \prime}
\end{aligned}
$$


On the other hand

$$
\begin{aligned}
\sum^{\prime} & \leq C \sum_{n=0}^{\infty} \frac{n^{2(2 \alpha+3-s)+1}}{R^{4 n}} \int_{\Omega}|z|^{2 n} \omega(z)^{\alpha} d m(w) \\
& \leq C \sum_{n=0}^{\infty} \frac{n^{3 \alpha+6-2 s}}{R^{2 n}}|w|^{2 n} \leq C \sum_{n=0}^{\infty} \frac{|w|^{2 n}}{R^{2 n}} \\
& =C\left(1-\frac{|w|^{2}}{R^{2}}\right)^{-1} \leq C \omega(w)^{-2(\alpha+2)},
\end{aligned}
$$

and the same holds for $\Sigma^{\prime \prime}$ (see Section 4). So we have

$$
\left\|u_{s w}\right\|_{\alpha}^{2} \leq C \omega(w)^{-(\alpha+2)} \text {. }
$$

Similarly, we can work out the weak factorization for $L_{-}$. Then we have proved the theorem.

The $S_{p}$-results for Hankel forms then follows immediately, more or less as we have done before.

THEOREM. For $1<p \leq \infty$, the Hankel form $\Gamma_{b}$ is in $S_{p}$ iff $b \in A^{\alpha^{\prime}, p}(\Omega)$.

\section{REFERENCES}

1. F. A. Berezin, General concept of quantization, Commun. Math. Phys. 40 (1975), $153-174$

2. R. G. Gunning, Lectures on Riemann surfaces (Princeton University Press, 1966).

3. B. Gustafsson and J. Peetre, Notes on projective structures on complex manifolds, Nagoya Math. J. 116 (1989), 63-88.

4. B. Gustafsson and J. Peetre, Hankel forms on multiply connected domains. Part Two. The case of higher connectivity, Complex Variables 13 (1990), 239-250. 205-219.

5. S. Janson, Hankel operators between weighted Bergman spaces, Ark. Mat. 26 (1988),

6. S. Janson, J. Peetre and R. Rochberg, Hankel forms and the Fock space, Revista Mat. Iberoamericana. 3 (1987), 61-138.

7. J. Peetre, Hankel forms on multiply connected domains. Part One. The case of connectivity two, Complex Variables 10 (1988), 123-139. $165-186$.

8. J. Peetre, The Berezin transform and Ha-plitz operators, J. Operator Theory 24 (1990),

9. J. Peetre, New Thoughts on Besov spaces (Duke University Mathematics Series 1), Duke University, Durham, 1976.

10. J. Peetre and J. Karlsson, Rational approximation-analysis of the work of Pekarskiri, Rocky Mountain J. Math. 19 (1989), 313-333.

11. R. Rochberg, Decomposition theorems for Bergman spaces and applications, in: Operators and Function Theory (ed. S. C. Power), Proceedings, Lancaster 1984, p. 225-277. Reidel, Dordrecht, 1985.

12. R. Rochberg and S. Semmes, A decomposition theorem for functions in BMO and application, J. Functional Analysis 67 (1986), 228-263.

Matematiska InStitutionen, STOCKHOLMS UNIVERSITET, Box 6701, S-113 85 STOCKHOLM, SWEDEN 